\title{
Identification of Topological Spin Textures in Frustrated Fe3Sn2 Magnetic System
}

Jiang Cui ${ }^{1}$, Hongkui Zheng ${ }^{1}$, John $\mathrm{Watt}^{2}$ and $\mathrm{Kai} \mathrm{He}^{3}$

${ }^{1}$ Clemson University, United States, ${ }^{2}$ Los Alamos National Laboratory, Connecticut, United States,

${ }^{3}$ Clemson University, Clemson, South Carolina, United States

Since the groundbreaking discovery of intrinsic ferromagnetism in atomically thin two-dimensional (2D) van der Waals (vdW) $\mathrm{Cr}_{2} \mathrm{Ge}_{2} \mathrm{Te}_{6}$ and $\mathrm{CrI}_{3}$ materials [1, 2], there has been intensive interest in identifying and understanding the topological spin textures in $2 \mathrm{D} \mathrm{vdW}$ systems. It is expected that topologically nontrivial spin textures can be excited from the spin-polarized ground state. However, only a handful of studies have so far been reported to visually identify the skyrmion-like quasiparticle states using Lorentz transmission electron microscopy (TEM) [3-6]. Despite the recent progress in this field, the knowledge about the detailed correlations between the intrinsic topological spin characters and the extrinsic tuning factors, such as lattice geometry and the number of vdW layers, still remains poorly understood and less explored.

In a specific 2D vdW magnetic system, $\mathrm{Fe}_{3} \mathrm{Sn}_{2}$ possesses layered Kagome lattice, which induces the geometrical frustration in correlative spin alignments. It has been found that such unique spin-orbit and spin-lattice coupling can give rise to topological skyrmion spin textures at finite temperature and magnetic field $[7,8]$. Here, we aim to use cryogenic Lorentz TEM to investigate the spin textures of $\mathrm{Fe}_{3} \mathrm{Sn}_{2}$ at low temperatures. In order to reveal the dependence of the number of vdW layers, we have prepared the Fe3 $\mathrm{Sn}_{2}$ sample into different controlled thicknesses (i.e., 40, 60, and $80 \mathrm{~nm}$ ) using the focused ion beam (FIB) technique, as shown in Figure 1a. The as-prepared $\mathrm{Fe}_{3} \mathrm{Sn}_{2}$ sample is a high-quality single crystal, as confirmed by high-resolution TEM (HRTEM) and selected area electron diffraction (SAED), shown in Figure $1 b$ and $c$. With the given intrinsic magnetocrystalline anisotropy and the same thickness the FIB lamella, we expect that the room-temperature stable stripe domain appear in the identical domain width. However, our Lorentz TEM observation (Figure 1d) indicates that two types of stripe domains with distinct domain widths were formed within the same $40 \mathrm{~nm}$ thick slab of the FIB sample. This unexpected result may imply that some external tuning factors could show the predominant impact on the formation of spin textures, which may also influence the topological characters of skyrmion-like excitation states. Further investigation is needed to validate this hypothetic question and clarify the underlying mechanism for the fundamental spin-orbit coupling in 2D vdW systems [9].
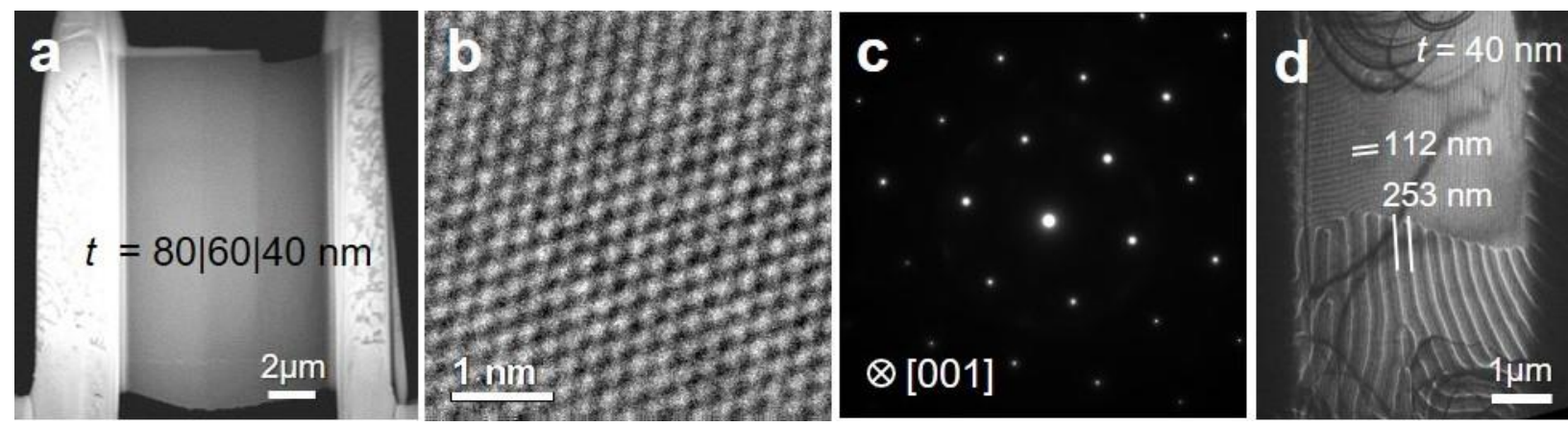
Figure 1. Figure 1. (a) FIB sample of Fe3Sn2 with varied thickness of $40 \mathrm{~nm}, 60 \mathrm{~nm}$, and $80 \mathrm{~nm}$. (b) HRTEM image and (c) SAED pattern of Fe3Sn2 sample. (d) Lorentz TEM image of $40 \mathrm{~nm}$ thick Fe3Sn2 showing the stripe domain structures with different domain widths.

\section{References}

[1] C. Gong et al., Nature 546, 265 (2017).

[2] B. Huang et al., Nature 546, 270 (2017).

[3] M. G. Han et al., Nano Lett., 19, 7859 (2019).

[4] Y. Wu et al., Nature Commun. 11, 3860 (2020).

[5] B. Ding et al., Nano Letter., 20, 868 (2020).

[6] M. Yang et al., Sci. Adv., 6, eabb5157 (2020).

[7] Z. Hou et al., Adv. Mater., 29, 1701144 (2017).

[8] Z. Hou et al., Adv. Mater., 32, 1904815 (2020).

[9] The authors acknowledge the funding support by National Science Foundation (Award \#1929138) and the use of Electron Microscopy Facility at Clemson University. This work was performed, in part, at the Center for Integrated Nanotechnologies, an Office of Science User Facility operated for the U.S. Department of Energy (DOE) Office of Science by Los Alamos National Laboratory (Contract 89233218CNA000001) and Sandia National Laboratories (Contract DE-NA-0003525). 